TIPA. Travaux interdisciplinaires sur la parole et le langage

$34 \mid 2018$

La langue des signes, c'est comme ça

\title{
Traduire la poésie en langue des signes : un défi pour le traducteur
}

Translation and signed poetry: a challenge for the translator

Pénélope Houwenaghel and Annie Risler

\section{OpenEdition}

1 Journals

\section{Electronic version}

URL: http://journals.openedition.org/tipa/2384

DOI: 10.4000/tipa.2384

ISSN: 2264-7082

\section{Publisher}

Laboratoire Parole et Langage

\section{Electronic reference}

Pénélope Houwenaghel and Annie Risler, «Traduire la poésie en langue des signes : un défı pour le traducteur », TIPA. Travaux interdisciplinaires sur la parole et le langage [Online], 34 | 2018, Online since 20 September 2018, connection on 09 October 2020. URL : http://journals.openedition.org/tipa/2384 ; DOI : https://doi.org/10.4000/tipa.2384

This text was automatically generated on 9 October 2020 .

La revue TIPA. Travaux interdisciplinaires sur la parole et le langage est mise à disposition selon les termes de la licence Creative Commons Attribution - Pas d'Utilisation Commerciale - Pas de Modification 4.0 International. 


\section{Traduire la poésie en langue des signes : un défi pour le traducteur}

Translation and signed poetry: a challenge for the translator

Pénélope Houwenaghel and Annie Risler

\section{0 . Une question d'inter-culturalité}

1 Les Sourds du monde entier forment une communauté sans territoire : une diaspora. L'usage de S majuscule, et du mot Sourd s'est imposé, pour désigner les sourds en tant que personnes, en tant que réalité sociale ou anthropologique (Mottez, 1993). Le sociologue peut effectivement parler d'une communauté Sourde, qui partage une culture et une langue communes. La culture Sourde résulte de plusieurs éléments conjugués dont, en premier lieu, un mode d'être au monde spécifique, basé sur la culture du visuel. La sensation, l'impression visuelle prime. La réalisatrice et productrice, Sandrine Herman illustre la primauté de la perception visuelle chez les Sourds par une anecdote, qu'elle relate dans le documentaire Sourds et malentendus (Ochronowicz, 2009). Elle se souvient que, petite, elle percevait la pluie comme une fête. La perception de la multitude d'impacts de gouttes dans les flaques est pour elle ce qui s'apparente à un chant ou une musique.

2 La culture Sourde s'identifie en second lieu par un vécu partagé, des coutumes, un folklore, des manifestations de tous ordres comme des banquets, des rassemblements sportifs (au premier rang desquels se trouvent les Deaflympics, également appelés Jeux Olympiques des Sourds), l'existence de lieux de rencontres informels, des foyers, etc.

3 La culture Sourde, enfin, peut être définie comme l'appartenance à une communauté Sourde, bien plus qu'une identité Sourde. Mottez (1987) met en évidence que la fréquentation des lieux de socialisation Sourde amène l'individu à prendre conscience de sa propre identité. C'est en effet dans ces lieux où la surdité peut être oubliée, annulée, puisqu'elle n'est plus source de discriminations ou de difficultés de communication, que le Sourd peut développer sa personnalité. Cette communauté 
mondiale est structurée aussi bien sur le plan local, national, transnational et mondial, à travers nombre d'associations, d'amicales, de clubs, de fédérations.

4 Cette communauté Sourde exprime son rapport au monde par des pratiques artistiques au premier rang desquelles la littérature signée, ou performance signée, dont nous allons parler ici. Il existe réellement tout un patrimoine qui s'enrichit en permanence de formes nouvelles, dont on peut depuis quelques décennies garder trace à travers la vidéo. Le développement des supports audiovisuels a libéré les formes créatives en langue des signes. En effet, la démocratisation des supports analogiques puis numériques a favorisé la diffusion des œuvres signées, puis propagé à l'échelle mondiale les créations d'artistes. Ces formes artistiques culturellement marquées portent un discours, et sont traversées par une parole signée. Elles interpellent d'autres milieux culturels, comme le nôtre (milieu entendant de différentes langues).

Comme le précise Newmark (1981), la traduction fait partie intégrante de la vie intellectuelle de tout peuple civilisé. La communauté Sourde ne fait pas exception. Elle « importe» des traductions dans son territoire culturel et « exporte» des traductions. Se pose donc très clairement la question de la traduction, en tant que passage d'un texte d'une culture à une autre. La traduction permet de transmettre des informations à des locuteurs de langues différentes. En ce sens, ainsi que l'écrit Peeters (1999), le traducteur est un acteur sociolinguistique chargé de pallier la rupture d'intercompréhension entre des interlocuteurs mutuellement étrangers.

Nombre de questions se posent au chercheur : sommes-nous face à un cas particulier de traduction? Qui traduit ces œuvres signées? Dans quel but? Comment? Peut-on répertorier et quantifier les traductions réalisées dans un sens et dans l'autre? Quels impacts ont ces travaux? Pour essayer d'avancer sur ces différentes questions, nous devrons d'abord, dans une première partie, revenir sur le matériau qui s'offre à la traduction, à savoir le texte littéraire. Cette dénomination n'est en effet pas sans poser d'autres questions, quand on l'applique à la langue des signes. L'existence de nombreuses formes littéraires en langue des signes est attestée, par exemple les œuvres couvrant les champs du théâtre, de l'humour, la poésie ou encore le chansigne. Ces diverses formes artistiques englobent différents aspects. Mais dans tous les cas, du fait que la langue des signes emprunte une modalité linguistique et expressive à partir des mouvements du corps, il y a d'emblée une difficulté à délimiter ce qu'on va appeler le texte.

\section{La poésie signée : une forme littéraire, artistique?}

\subsection{Qu'est-ce qu'un texte en langue des signes?}

7 Le point de vue linguistique permet de mesurer la complexité de cette question. Identifier un texte amène à en déterminer les contours, les limites. Et d'une certaine manière, à s'interroger sur les limites entre ce qui est linguistique et ce qui ne le serait pas. Nous allons voir que c'est d'autant plus compliqué que les langues des signes sont des langues de l'oralité, du face-à-face, et qu'elles s'expriment dans une modalité visuogestuelle.

8 Une langue des signes n'ayant pas de forme écrite avec un alphabet, sur papier, on a donc affaire à des textes oraux. Les langues signées sont des langues de l'oralité. Elles partagent plus de caractéristiques avec la forme orale du langage que sa forme écrite. 
De fait, l'oralité implique toujours à la fois du verbal, du para-verbal et du non-verbal. Le para-verbal concerne les phénomènes intonatifs et rythmiques. Le non-verbal regroupe les gestes manuels, les gestes corporels comme les changements de postures, les mimiques, et les regards. Le registre oral des langues est donc multimodal. Fonagy (1983) ou Bouvet \& Morel (2002) signalent que les gestes et la mimique accompagnent en permanence la parole, comme une deuxième voix. De grands débats agitent la communauté scientifique aujourd'hui, qui cherche à établir les liens entre les deux systèmes sémiotiques que sont la langue et la gestualité. Si Goldin-Meadow (2003) insiste sur l'aide qu'apporte le geste à la parole, en affirmant que les gestes aident à penser, Kendon (2008) montre que gestes et mots ne donnent pas toujours les mêmes informations mais contribuent tous deux à la construction du sens. Ce sont deux systèmes sémiotiques différents, mais complémentaires qui interagissent et sur lesquels s'appuie conjointement tout locuteur. Le sens ne repose en effet pas uniquement sur les mots et leurs combinaisons. Pour Guaïtella (2013), il n'y aurait d'ailleurs pas d'interaction langagière possible sans le soubassement de la gestualité, sur laquelle viennent se poser les mots comme des compléments informatifs.

Une langue des signes a comme caractéristique d'être une langue visuo-gestuelle. Cela signifie qu'elle s'articule avec toutes les parties du corps: le mode de signification résulte de mouvements des bras et des mains, de postures, de mimiques, de regards. Le matériau linguistique prend la forme d'un tracé complexe dans l'espace, constitué par la combinaison à la fois temporelle et spatiale des différents éléments signifiants. Le corps, le visage et le regard du signeur sont toujours offerts à la vue. Les « mots » sont articulés par des mouvements manuels, codifiés. Mais en langue des signes, les suites de mots doivent se combiner aussi bien séquentiellement que spatialement pour construire du sens. Chaque élément auquel réfère le signeur se trouve associé à un emplacement, et le prédicat a comme fonction de mettre littéralement en relation les différents objets du discours. Le corps du signeur lui-même constitue le point d'origine à partir duquel sont calculées toutes les références énoncées et disposées dans l'espace autour de lui, qu'il s'agisse de références personnelles, temporelles ou locatives. Quand le signeur prend une mimique de douleur, de surprise, de dénégation, cela ne signifie pas qu'il a mal, qu'il est surpris ou qu'il n'est pas d'accord, mais qu'il fait référence à la douleur de quelqu'un, qu'il introduit un doute dans son discours ou une marque de négation. Quand il pointe l'index dans une direction, il ne désigne pas un élément présent dans l'espace physique qui l'entoure (le sol, le mur), mais il réfère à un élément antérieur de son discours associé à un emplacement. Ainsi que le rappelle Kendon (2008), les gestes à valeur linguistique des signeurs ne sont donc pas à prendre au premier degré, qu'il s'agisse de gestes de désignation, de postures ou de gestes d'actions. Le regard du signeur acquiert un statut de marqueur linguistique, tout autant que les changements de posture, même s'ils sont similaires aux regards et postures des locuteurs en langues vocales. De même pour les gestes de désignation ou les mimiques.

C'est pourquoi on parle de langue incarnée: toute action, sensation, perception est exprimée par un mouvement qui part du corps du signeur, qu'il parle de lui ou d'un ou plusieurs autres (Risler, 2016b). Le signeur opère en permanence des changements de perspective qui l'amènent à associer la position et les mouvements de son corps avec la position et les mouvements de celui dont il parle. Ces prises de rôle, qui provoquent les changements de perspective, accompagnent la progression discursive à travers les actions, perceptions ou propos rapportés. Elles se manifestent par des changements de posture, de regard, de mimique, qui se retrouvent ainsi au cœur de la construction 
linguistique. Il ne s'agit pas simplement de marques d'expressivité, de gestualité para ou co-verbale.

11 La modalité visuo-gestuelle des langues signées chamboule donc complètement les limites entre le verbal, le para-verbal, le non-verbal. Avec les langues vocales, il est relativement plus aisé de faire la part entre les trois, en distinguant les mots des accents, du rythme, de l'intonation, de la gestualité (mais peut-être cela tient-il au fait qu'on en ait plus l'habitude ?). Pour les langues des signes, tout est ramené à la seule modalité gestuelle, support de tous les éléments échangés lors de l'interaction langagière : le verbal, et le non-verbal, à travers les mouvements du corps, de la face, du regard ou les gestes manuels. Peut-on encore faire la part du verbal et du nonverbal ? Cormier (2014) fait remarquer que les linguistes des langues signées ont longtemps eu tendance à considérer que toute forme expressive d'un signeur est une forme linguistique. Elle associe cette attitude peu rationnelle à une volonté inconsciente de "réparer » le déni de l'existence de langue des sourds qui a perduré jusqu'à la fin du vingtième siècle. Risler (2016a) ajoute que la tendance a été renforcée par le fait que les linguistes se sont massivement appuyés sur des discours narratifs et descriptifs pour décrire le fonctionnement des langues des signes. Or la part du sens portée par la gestualité, laquelle intervient dans tous les contextes d'oralité en face à face, se trouve accentuée encore dans ces situations où le locuteur cherche à théâtraliser plus particulièrement son propos.

Gestualité et langage se combinent d'autant plus dans les registres de littérature orale, parce que s'ajoute une intention manifeste de donner à voir et à entendre. Par son jeu corporel, le conteur a la possibilité d'incarner celui dont il parle ou de simuler des mises en espace des objets de son discours, en utilisant ainsi au maximum les effets de sens apportés par la gestualité, la mimique et la voix. Il en est de même pour le conteur signeur, qui peut stylistiquement, dans une intention illustrative de donner à voir, renforcer son jeu corporel et ainsi maximiser les effets de sens apportés par la gestualité et la mimique. Ce registre de discours ne favorise donc pas la tâche des linguistes qui essaient de faire la part entre les constructions verbales et les effets nonverbaux.

Les approches de la multimodalité des langues orales amènent ainsi à relativiser l'omniprésence du verbal, dans les registres aussi bien narratifs que littéraires, en langues des signes. La multimodalité (propre à la littérature orale ainsi qu'à toute forme d'interaction en langue orale) se marque, pour les langues des signes, dans un recours enrichi et complexifié à la gestualité. Ainsi, le jeu de toutes les parties du corps du signeur est mis au service à la fois du jeu corporel - c'est-à-dire de l'expressivité corporelle - et des prises de rôles ${ }^{1}$ - ou marquage linguistique des changements de perspective énonciatives induites par la structure-même de la langue des signes (Risler, 2016b).

Dans la performance corporelle se mêlent et s'entremêlent, sans qu'on sache encore aujourd'hui les délimiter, la part verbale, non-verbale et para-verbale. C'est là un nouvel enjeu de la recherche aussi bien linguistique que littéraire : où commence et où s'arrête le texte? On verra que ce sont des questions que ne se posent pas les auteurs, mais que devront se poser les traducteurs. 


\section{2. La poésie signée : de la création signée au sign-art}

15 La poésie signée soulève de nombreuses questions de recherche, quant à sa nature même, ce qui la constitue, mais aussi quant à la terminologie employée pour la désigner, la façon de la décrire et de la comprendre, sa perception esthétique, sa résonnance avec les expressions artistiques actuelles, tout autant que sa genèse, son histoire, la place qu'elle occupe actuellement dans les communautés Sourdes et ce qu'elle dit de ces communautés et de leur rapport au monde. Julie Chateauvert (2014) a problématisé une partie de ces questions dans sa thèse de doctorat en Études et pratiques des arts, intitulée «Poétique du mouvement: ce que les langues des signes font à la littérature ». Elle a cherché à aborder la création signée du point de vue de la critique littéraire de ces œuvres, champ peu exploré et qui nécessite la création d'outils.

L'œuvre d'art, comme la définit Huyghe (1955), est

une image, représentative ou expressive [...] de ce que [l'homme] perçoit en lui, à l'aide analogiquement de ce qu'il perçoit autour de lui; mais cette image pour accéder à l'art, doit se constituer en un tout organisé et indépendant, n'ayant d'autre but que son propre accomplissement.

17 L'œuvre naît de la vision d'un individu, de sa singularité d'être au monde, l'œuvre est un partage. Elle est avant tout un produit intellectualisé, «médiumisé » et ressenti. Ce que nous en voyons et en saisissons n'est qu'une perception passée au filtre de nos propres représentations. Dans le cas des œuvres vectorisées par les langues des signes, l'œuvre est une performance du corps en mouvement. Le lecteur (averti ou non) est un spectateur.

Les premiers travaux sur les créations signées s'attachent à classifier les productions, à nommer les formes observées. Nancy Frishberg en 1988 est la première à proposer une classification des œuvres signées. Elle distingue trois genres: oratoire, folklorique (humour, narrations, histoires à contraintes de formes manuelles) et performance art qui recouvre la poésie, les formes narratives préparées et répétées dans le but d'être montrées à un public. Elle procède à une catégorisation assez large se basant sur la nature des textes. Ben Bahan, en 2007, actualise cette classification en proposant trois catégories : les chansons, les formes narratives et les histoires avec contraintes de configurations manuelles (Bahan, 2007). La classification par type de texte, en se référant à la littérature orale, permet de faire la part entre la poésie, forme courte, plus ou moins narrative; le récit, forme narrative longue; le théâtre; le chant-signe (chansigne) $)^{2}$, équivalent signé d'une chanson; et le folklore : histoires drôles, blagues, proverbes.

La poésie contemporaine, qu'elle soit vocale ou signée fait voler en éclat les catégories : on range donc sous ce vocable toutes les formes courtes, alors qu'elles n'ont pas toujours grand-chose à voir entre elles. Les auteurs ont toujours essayé de repousser la limite du verbal, de la langue dans le domaine littéraire. En témoignent par exemple les mises en forme graphiques, le jeu sur les sons, les bruits, les mots, la musicalité, le rythme. De la même manière, la création littéraire, ou création artistique dans une langue signée ne se limite pas à du texte. Du fait de ce mode d'expression gestuel, il y a, dès le départ, du registre littéraire, narratif ou poétique, une imbrication très étroite entre des effets de langue, de style, d'image, de ressenti lié à la présence de celui qui 
produit l'œuvre, la gestualité, en un mot à la performance qui mêle langue, jeu corporel et chorégraphie.

En 1993, Clayton Valli, linguiste et poète Sourd américain, propose une classification de la poésie signée en quatre catégories : les poésies écrites en anglais par des sourds, les traductions littérales (mot à signe) de poésies écrites en anglais, les traductions à proprement parler et les poésies créées directement en langue des signes. Il montre que ces catégories se juxtaposent chronologiquement. Cependant, on constate aujourd'hui, d'une part que les artistes Sourds investissent simultanément une ou plusieurs approches suivant leur appétence et leur mode d'expression privilégié, d'autre part que la création poétique signée s'étale de la poésie traditionnelle - qu'il conviendra de ranger dans le registre de la littérature orale - à la création multimédia.

Les langues des signes sont des langues de l'oralité, du face-à-face, qui n'ont pas de forme écrite. Cependant, les œuvres signées peuvent être conservées sous forme d'archive vidéo. Rose (1992) est une des premières à considérer qu'il y a un avant et un après VHS. Dans ce cas, la vidéo remplit un double rôle : d'une part comme moyen d'enregistrement et de conservation d'une performance (trace d'une expression particulière, d'une production orale instantanée et éphémère) ; d'autre part la vidéo comme performance multimédia en soi. La performance est fugace, elle disparaît si on ne l'enregistre pas. La vidéo permet ainsi la reproduction, la transmission, la conservation du patrimoine oral signé. Mais la vidéo peut aussi être le résultat d'un travail d'écriture. Il s'agit de la vidéo comme œuvre multimédia, dans laquelle l'auteur va mêler différentes techniques en rapport avec le médium choisi, ici l'image animée vidéo. L'œuvre devient alors un produit fini, diffusable, mais fixé. Ces œuvres sont bien de leur temps. A l'époque de l'avènement de l'image et de l'explosion des produits vidéo, les langues des signes trouvent un nouveau souffle. L'artiste Sourd d'aujourd'hui ne travaille plus seul. Il rencontre ses pairs, s'entoure d'une équipe technique (vidéaste, chorégraphe, développeur, communicant) et suivi par des fans sur les réseaux sociaux et les plateformes de vidéo en ligne.

Chateauvert (2014) soutient que la poésie signée constitue une forme artistique plus que strictement littéraire. Il y a une connexion étroite entre le langage du corps et la langue gestuelle - mais l'un ne se dissout pas dans l'autre! - entre un art du mouvement, la chorégraphie, le dessin dans l'espace, le jeu de rôle et le texte. Cela ferait de la poésie signée un art en soi. Pollit (2014) propose le terme anglais de sign-art dans la ligne du vidéo-art, du media-art, performing-art. Il est difficile de traduire ce terme en français, dans la logique de la langue française. Cependant, au-delà des questions de terminologie, l'œuvre d'art questionne le spectateur et le chercheur dans ses aspects formels. L'analyse de corpus d'œuvres signées révèlent plusieurs procédés qui peuvent être qualifiés de poétiques même si, comme l'écrit Thion, cette littérature signée adopte des formes très diverses, que les sourds eux-mêmes ne nomment pas toujours poésie (2015). Certaines techniques sont partagées par les créations en langues vocales, d'autres s'appuient sur la spécificité du mode d'expression visuo-gestuel de la langue des signes.

Parmi les procédés discursifs « classiques » ou universels, utilisés par les poètes Sourds, on relève les répétitions (Valli, 1993 ; Blondel, 2000), les rimes par paramètre (Valli, 1993), la fluidité ou le fondu entre les signes (Klima \& Bellugi, 1976), la symétrie (Sutton-Spence \& Kaneko, 2007), les métaphores (Taub, 2001; Delaporte, 1999), l'amplitude (Blondel, 2000). 

visual), ou exploitation maximale de la figurabilité du corps, de l'utilisation des différentes parties du corps pour figurer; et les contraintes paramétriques (jeux d'homophonies) à partir d'une configuration de la main, ou d'un mouvement, qui se retrouvent comme autant d'assonances. Le $\mathrm{VV}$, en jouant sur la proximité avec l'imitation, repose sur les mêmes moyens de figuration, de construction de références dans l'espace que les langues signées, mais il évacue toute utilisation du lexique conventionnel et standardisé. Au contraire, les contraintes paramétriques jouent sur la forme visible des articulateurs et la motivation des formes lexicales. Ces deux procédés font apparaître des contrastes très forts dans la part respective prise par le jeu corporel et chorégraphique et la forme linguistique, qui vont bien au-delà de l'effet de style. leur nature, leur conception, et à fournir une vision globale et consensuelle, des traducteurs s'emparent de ces œuvres et les traduisent bel et bien dans d'autres langues. Les questions des chercheurs recoupent-elles les problématiques que soulève la traduction d'œuvres signées?

\section{Pourquoi la traduction ? Comment traduire la performance signée?}

Les nombreuses questions qui se posent sont en lien avec la limite du texte, les concepts d'œuvre, d'auteur, de transmission. Appréhender une œuvre signée est complexe et contraint le traducteur à poser sur l'œuvre un regard polymorphe, s'attachant tout autant à saisir les contours plastiques de l'œuvre que ses aspects internes et externes. Nous nous intéresserons ici à la traduction de poésie en France. Si, comme l'écrit Genette (1982), le plus sage, pour le traducteur, serait sans doute d'admettre qu'il ne peut faire que mal, alors nombre de traductions littéraires n'auraient jamais vu le jour. Genette rappelle ainsi la perfectibilité des traductions et l'humilité qui anime les traducteurs.

\subsection{Les prémices de la traduction littéraire en langue des signes française}

Les prémices de performances poétiques signées en France apparaissent au début des années 1980, à l'International Visuel Theatre ${ }^{3}$, autour de Levent Beskardes, poète et comédien Sourd né en 1949. Il est une figure majeure de la scène poétique française. Un entretien, qu'il nous a accordé le 14 août 2017, nous permet de mieux comprendre son travail et illustre notre propos. À l'âge de 18 ans, il découvre la poésie dans son pays natal, la Turquie. Ses premières créations poétiques en langue des signes datent de la toute fin des années 1960, époque où la VHS ${ }^{4}$ n'existe pas. Il conserve la trace de ses poèmes en passant par l'écrit, en notant quelques mots en turc. Son poème intitulé " Rouge » est ainsi conservé grâce à une translittération ${ }^{5}$ en langue turque qu'il dit maîtriser très maladroitement et qui ne lui permet pas de richesse d'expression.

Devenu comédien, il s'essaye à la traduction de poèmes turcs évoquant la souffrance et l'emprisonnement. Il reconnaît qu'à l'époque il est loin de comprendre le sens réel des textes et qu'il ne transpose en langue des signes que sa perception partielle des textes, 
l'esthétique qu'il y projette et les rêveries qu'il y associe. En 1980, il s'installe en France, travaille à l'International Visuel Theatre (IVT), aux côtés d'Alfredo Corrado et Chantal Liennel. Ses traductions / adaptations de poésies turques sont accueillies avec enthousiasme par l'équipe de comédiens. Et pour cause, à cette époque en France les Sourds sont éloignés de toute forme de poésie. Ils n'ont accès qu'à des translittérations médiocres réalisées par des enseignants non-natifs de la langue des signes. L'IVT constitue alors le premier atelier de poésie en langue des signes de France. L'émulation qui y règne permet, notamment, à Chantal Liennel de créer le poème "Fleur " $^{6}$, directement en langue des signes française. Elle associe le geste-danse à la composition dans l'espace. Chantal Liennel réalise ensuite elle-même l'adaptation en français :

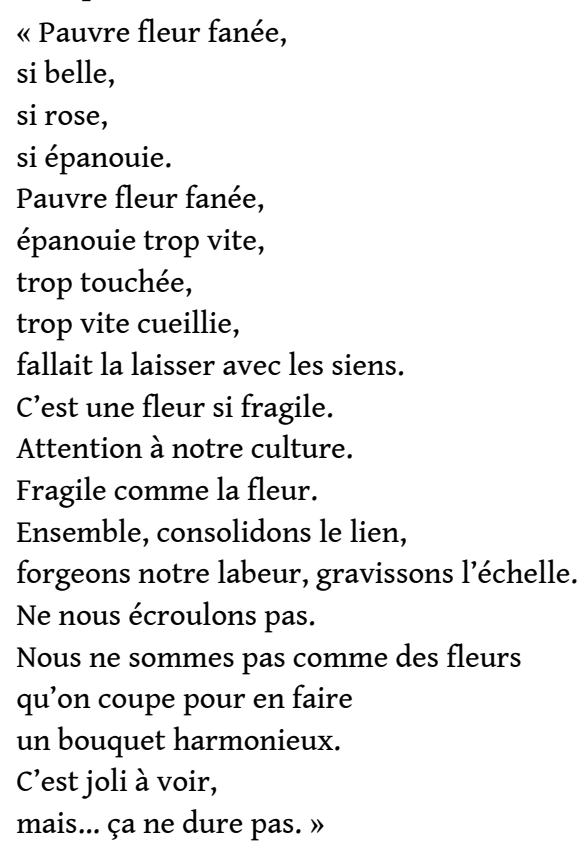

Ce poème, bien que connu des artistes Sourds de cette génération car symbole du renouveau et de l'éveil de la conscience communautaire des Sourds français à la fin des années 1970, reste difficilement accessible au grand public entendant ${ }^{7}$. Pourtant, nombre d'artistes Sourds le connaissent et peuvent en réciter quelques vers sous leur forme signée. Ce poème semble appartenir à la tradition orale de la communauté Sourde. Mais les supports vidéo manquent pour une transmission aux générations actuelles en tant qu'élément du patrimoine culturel Sourd et surtout une reconnaissance de celui-ci par le grand public.

Ces premiers éléments, portant sur la création de l'IVT, la création des premiers poèmes signés, la rencontre d'artistes Sourds en France, fixent des repères chronologiques qui attestent de la naissance d'un mouvement poétique Sourd en France au tout début des années 1980. Ils confirment que les poètes Sourds, comme de nombreux poètes de langues vocales à travers le monde, réalisent des traductions, soit de leurs propres œuvres vers la langue nationale dominante, soit traduisent en langue des signes des poèmes créés en langue vocale. Par ailleurs, on notera que l'interculturalité et l'émigration sur le territoire français d'un jeune poète sourd turc est à l'origine d'un mouvement artistique qui ne va cesser de prendre de l'ampleur en France à la toute fin du XXème siècle. 


\subsection{Traduction par un tiers poète}

31 Les traductions de poésies signées se multiplient au cours des années 2000. Différents processus de traductions sont expérimentés. L'un d'eux est la traduction par un tiers poète. L'exemple de traduction de poème signé réalisée par des poètes entendants que nous allons présenter rend compte des obstacles rencontrés. N'oublions pas, en effet, que faire revivre dans la langue d'accueil l'expérience vécue par le créateur du poème exige que l'on recrée ses modes de pensée, d'imagination et d'expression (Ellrodt, 2006).

Quand la compréhension est partielle, quand le traducteur ne perçoit pas l'œuvre originale dans son entièreté, la traduction reste imparfaite.

Pour exemple, Levent Beskardes relate la traduction vers le français, en 2002, de son poème « Rouge ", par des poètes entendants. Ce poème, initialement créé en langue des signes turque est totalement repris par son auteur qui le recrée dans sa langue d'adoption, la langue des signes française. Levent Beskardes organise un «laboratoire poétique » de cinq jours, réunissant deux poètes entendants qui ne connaissent pas la langue des signes et un interprète. Les deux premiers jours l'auteur signe son poème face aux deux poètes (en présence de l'interprète), sans expliquer quoi que ce soit du contenu. Puis il laisse les poètes travailler à leur version. Le résultat est mitigé. S'inspirant de ce qu'ils croient comprendre de la LSF, ils traduisent le signe [ROUGE] qui ponctue chaque strophe par le mot «bouche ». Pour comprendre la confusion, il faut savoir que le signe [ROUGE] se prononce par un mouvement de l'index sur la bouche. Les poètes-traducteurs se sont donc fiés à leur perception du geste de l'index sur la bouche. Or il s'agissait d'un signe conventionnel, figé en langue des signes, et non d'un élément non-verbal, montrer la bouche. La confusion vient de ce que le texte est ici perçu comme de la gestualité, du fait de la méconnaissance de la langue source. Même si Levent Beskardes loue les qualités indéniables du reste de cette traduction, le sens du poème en est totalement transformé. Les poètes revoient leur copie et les deux versions sont tout de même présentées conjointement en lecture publique. D'autres études de cas permettraient de mieux comprendre l'apport d'un traducteur, à condition que la connaissance imparfaite de la langue source ne soit pas un obstacle. Des travaux pourraient être menés dans cette direction.

\subsection{Traduction par l'auteur, le bilinguisme-biculturalisme}

A mesure que les poètes Sourds développent leur art et s'ouvrent à la littérature poétique d'autres cultures, ils produisent eux-mêmes des traductions. Les processus de traductions s'affinent et nous renseignent sur la forme du produit fini, le poème traduit dans la langue d'arrivée. Le chercheur dispose ainsi de plus en plus de matériaux, de corpus, pour comprendre la nature même de la poésie signée.

Les traductions de poèmes en langue turque, notamment ceux de Nazim Hikmet, réalisées par Levent Beskardes en langue des signes française permettent de saisir, audelà du bilinguisme, l'importance du biculturalisme. Rappelons ici que Levent Beskardes part d'une forme écrite, publiée, pour en faire un texte " oral ». Il s'appuie non seulement sur la version écrite turque, mais également sur une traduction en français, pour mieux saisir le sens. Ainsi, être biculturel à cheval entre la langue de son enfance (la langue des signes turque), sa langue d'adoption (la langue des signes 
française) et deux langues vocales (le français et le turc), il construit à partir de toutes ces langues sa perception et sa compréhension du poème original :

Je saisis le texte en turc mais n'en comprends pas le sens caché. Le français me permet de comprendre les subtilités (entretien, 2017).

Après avoir saisi les nuances du texte, il se détache de sa forme matérielle, de la forme verbale du poème. Il relate ainsi sa méthode :

J'invente des effets visuels, des jeux esthétiques, des néologismes. Je mets de côté la langue standard et cherche une forme libre, qui attirera le public.

Carlos Carreras, interprète, évoque des procédés similaires quand il travaille à la traduction vers le français d'une poésie en langue des signes :

C'est avant tout l'inspiration qui est sollicitée et elle varie en fonction des styles et des propositions. On va jouer sur différents paramètres, parfois on invente des mots car il y a des jeux de signes (notamment dans les traductions depuis des poésies de François Brajou) (Eckert-Poutot, 2016).

Ces deux témoignages confirment que, comme pour les langues vocales, les procédés employés en traduction depuis et vers la langue des signes sont identiques.

Levent Beskardes précise aussi qu'il adapte le poème à son public cible, les spectateurs Sourds. Pour ce faire, il sélectionne les éléments saillants et propose une adaptation. Ma priorité, ce sont les Sourds! défend-il quand il travaille à l'adaptation de poèmes vers la LSF. Il ne s'interdit pas de déplacer un vers ou une strophe pour que le public Sourd, éloigné de la littérature poétique, accède au sens du poème. Il se refuse à la traduction littérale, trop proche du texte d'origine qui perd de sa saveur et de son esthétique lors du passage vers la langue des signes. Il s'attache au sens et surtout à l'effet produit. La traduction, en tant que produit fini, doit être visuelle avant tout. Elle est produite pour être livrée sur scène, donnée au public.

D'autres poètes Sourds procèdent à la traduction de leur propre poésie, également dans une visée scénique. Djenebou Bathily, poétesse et comédienne Sourde française, assume pleinement la création d'œuvres à partir de deux langues ${ }^{8}$. Elle crée poésies et slam indifféremment en langue des signes ou en français. Elle s'intéresse particulièrement au passage entre un texte écrit et un poème signé. Pour le festival Voix Vives de Sète, elle monte elle-même sur scène et signe ses poésies en public, pendant que la traduction en français, qu'elle a elle-même réalisée, est lue par une comédienne. Pour le festival du film de Douarnenez en 2011, elle performe un slam dans le cadre d'une œuvre collective avec le groupe de musique Viscore. Le texte signé est joué en complémentarité avec la vidéo et la musique. Derrière la slameuse, un écran projette des images qui se transforment en mots.

Pour ces deux poètes, le texte et la performance scénique sont donc intimement liés. Ils ne constituent pas des cas isolés, puisque d'autres artistes mêlent texte et performance, notamment Jules Turlet, Ludovic Lortet ou Mathilde Chabbey. La traduction d'un texte écrit vers une langue signée va bien au-delà du passage d'un texte à l'autre.

\subsection{La traduction en binôme poète et interprète en langue des signes française/français}

D'autres procédés de traduction peuvent être observés. Certains poètes Sourds, y compris parmi ceux mentionnés plus haut, travaillent en binôme avec un interprète diplômé pour traduire leurs œuvres signées ou pour adapter des textes écrits. 
Travailler avec des interprètes leur permet de lever la barrière de la langue. Comme leurs homologues de langues vocales, les interprètes langue des signes française / français sont avant tout formés à l'interprétation. Ils sont aguerris au passage d'une langue à l'autre dans des situations d'expression orale, dans la simultanéité des interactions. La traduction, en tant que production d'une forme (soit écrite, soit enregistrée) à partir d'un texte écrit ou d'un texte signé, n'est pas leur cœur de métier. Cependant, on observe que certains collaborent avec des poètes Sourds. Même s'ils continuent alors à se nommer eux-mêmes interprètes, ils réalisent à ce moment-là un travail de traducteur.

Dans un premier cas de binôme poète Sourd / interprète, le poète est lui-même également traducteur diplômé. François Brajou, jeune poète de la scène contemporaine, s'est formé à la traduction au CETIM ${ }^{9}$ à Toulouse. Il participe lui-même à la traduction réalisée avec des interprètes, en intervenant sur le fond et la forme. Il affectionne ce type de collaboration, comme le montre son travail pour les éditions Benjamins média. Il réalise la traduction de plusieurs albums pour la jeunesse entre 2013 et 2017 : Un frère en bocal, Mimine et Momo, Le papa maman. La traduction ici n'est plus destinée à la scène mais s'inscrit dans une production multimédia pour le jeune public.

Dans un second cas, le poète s'adjoint les services d'un interprète qu'il choisit soigneusement. C'est le cas de Levent Beskardes. Il a notamment travaillé avec Laetitia Benasouli à la traduction de nombre de ses poèmes. L'un d'eux, «Âme » est édité en DVD par l'association Signes. Au-delà de ce travail permettant de conserver la trace du patrimoine poétique Sourd, Levent Beskardes reconnaît que son travail poétique est destiné à la scène. Il collabore actuellement avec Aurore Corominas, comédienne de formation et diplômée en interprétation français / LSF. Sollicité par un metteur en scène, il prépare la traduction de poèmes de Francis Ponge. Il décrit un travail de traduction difficile mais exaltant, éloigné des sujets qu'il aborde habituellement. La complexité de l'œuvre de Francis Ponge pousse le poète à adapter encore son processus de traduction.

La première étape est réalisée par l'interprète. Aurore Corominas, décrit Levent Beskardes, entame un travail de longue haleine de lecture pour saisir l'essence du texte. Le metteur en scène se positionne en ressource pour éclairer le sens du texte. Ensuite, elle opère une première traduction à destination du poète Sourd qui s'en imprègne. La deuxième étape peut alors commencer, le poète part de cette traduction pour produire une version en langue des signes.

L'interprète intervient donc au début du processus en tant que traductrice, au sens où elle travaille à partir du poème écrit, pour assurer au poète une compréhension fine du texte en français. Elle assure la traduction du français vers la LSF, de l'écrit vers une forme signée. Puis elle laisse le poète produire une version scénique. Cette dernière version est orale - au sens de produite en langue des signes française, langue ne disposant de trace écrite - idiomatique et scénarisée. Il y a un double mouvement : de la littérature écrite à une version en langue des signes, puis de la version signée à une forme orale. La version intermédiaire appartient-elle à la littérature écrite et orale ? Probablement est-elle déjà une version orale puisqu'elle est produite face au poète, comme une interprétation consécutive réalisée après de longues recherches.

La troisième étape s'enclenche ensuite. Aurore Corominas travaille avec le metteur en scène, cette fois-ci en tant que comédienne, pour ajuster la performance scénique en 
français. Enfin, les poèmes sont donnés publiquement à deux voix, en deux langues par le poète et la comédienne. Elle s'adapte à mon style, indique-t-il, elle module sa voix et saisit le public. C'est très émouvant. Cela fonctionne très bien. En filigrane, il nous dit ici la confiance qu'il accorde à la comédienne et surtout la qualité de leur relation, condition déterminante d'une traduction poétique réussie. De surcroît, on observe que la poésie signée se rapproche, ici encore, de la performance scénique. Une traduction réussie s'accompagnerait d'une confrontation au public, d'une représentation qui ne peut être réduite à une lecture aseptisée du texte.

\subsection{La traduction en équipe pluridisciplinaire : terrain d'expérimentation du festival Voix Vives} mobilisant un binôme poète / interprète, nous présentons ici un dernier dispositif : le travail en équipe pluridisciplinaire. Ce dispositif original est utilisé depuis une dizaine d'années dans le cadre du festival de poésie "Voix vives, de Méditerranée en Méditerranée ", dans son édition sétoise. Le festival connait également des éditions à Tolède, Gênes et Zakynthos (Grèce). Ce festival, consacré à la poésie et à l'interculturalité entre les peuples de tout le bassin méditerranéen, propose depuis presque dix ans une scène dédiée à la langue des signes française. Les travaux de traductions sont menés en partenariat avec l'association Arts Résonances, qui est à la fois un lieu d'échanges, de création, d'expérimentions et de recherches sur la poésie signée.

Dès 2011, les poètes Brigitte Baumié et Michel Thion invitent Levent Beskardes au festival Voix Vives. Il expérimente le travail avec une équipe mêlant interprètes et poètes. C'est le début d'une longue collaboration qui prend la forme d'une aventure collective. Carlos Carreras, interprète engagé dans cette collaboration dès sa création, confirme que l'idéal est de travailler en équipe avec plusieurs compétences réunies: linguistiques, artistiques, etc. (cité par Eckert-Poutot, 2016). Il ajoute que le travail est long quand il s'agit de réfléchir collectivement, traducteurs et poètes sourds et entendants réunis (ibid.). L'équipe compte des interprètes, spécialistes des langues dans leurs formes orales (vocales et signées), des traducteurs, spécialistes du passage d'une langue à l'autre à partir d'un texte écrit, des professeurs de langue des signes, un ou plusieurs poètes sourds ou entendants. La composition de l'équipe variera suivant les éditions. en fonction de son inclination et quelques poèmes de celui-ci. Le début du processus de traduction est donc individuel et suit le penchant naturel des traducteurs. Une semaine avant le festival, l'équipe pluridisciplinaire se réunit pour un "laboratoire poétique ", une session intense de mises en commun et de confrontations des propositions de traduction.

51 Les traductions de textes écrits vers une langue signée ouvrent un champ d'interrogations à l'équipe du festival : comment traduire? Comment rendre l'effet produit par le poème initial ? Comment rester fidèle au sens tout en conservant les effets poétiques? Comment trouver des équivalents aux effets sonores des poésies créées en langues vocales? Marie Lamothe, interprète de la première heure du festival Voix Vives, témoigne, au cours d'un entretien qu'elle nous a accordé en 2017, de la part d'inachevé d'une traduction :

TIPA. Travaux interdisciplinaires sur la parole et le langage, 34 | 2018 
Nous assumons notre trahison sans trop de culpabilité. Nous acceptons que dans notre proposition tout ne peut pas être présent (par exemple les sonorités) mais nous cherchons d'autres outils pour la perception du public.

nouvelles voix. En effet, Levent Beskardes s'amuse à créer parfois des poèmes qu'il sait destinés au festival et donc à la traduction. Il avoue créer volontairement des poèmes impossibles à traduire, pour éviter la traduction, tels des pièges pour les traducteurs. Il s'émerveille cependant de leur capacité à réussir tout de même à traduire ses œuvres. Son poème intitulé « V » fait justement partie de ces poèmes-pièges. Il utilise, lui aussi, une rime par paramètre, une seule configuration manuelle pour tout le poème : la lettre V. Pas moins de deux traducteurs, opérant depuis et vers la LSF, et deux poètes natifs du français travaillent à cette traduction. De leurs difficultés naîtront de nombreux néologismes (dansevibrer, danseflouer, valciller, viregraffer, etc.) pour rendre l'allitération d'origine.

\section{Traduire une forme vivante : à la limite du texte et de la performance signée}

\subsection{D'une langue à l'autre, d'un registre à l'autre}

57 Au-delà de la méthodologie et des dispositifs mis en place pour préparer le festival, des questions linguistiques se posent: quelle est la nature du texte source et du texte cible ? Le poème signé relève-t-il de la littérature orale? Traduire un poème signé vers 
le français, langue disposant d'une trace écrite, consiste-t-il à adapter de la littérature orale en littérature écrite ? Quel est le chemin à parcourir pour passer d'un texte écrit dans une langue à un texte oral dans une autre langue? Deux difficultés - ou deux défis - se présentent à l'équipe des poètes et traducteurs : le changement de registre et le changement de langue.

En ce qui concerne le changement de registre, Baetens (2016) résume bien la difficulté de transcrire un énoncé oral :

la transcription d'un énoncé oral n'est jamais le double visuel d'une production vocale, car les systèmes de notation habituels omettent un certain nombre de paramètres essentiels comme l'intonation, l'accent, la vitesse, le type de voix et ainsi de suite.

Une partie des effets et phénomènes nécessairement présents à l'oral ne sont pas forcément retranscrits à l'écrit : ce sont les éléments paraverbaux (prosodie, rythme, pauses, les particularités de l'orateur, les accents) et non-verbaux (gestes, mimiques postures). Certains peuvent être rendus à l'écrit, notamment par la ponctuation, des adaptations typographiques, des écritures pseudo-phonétiques, etc. Mais l'expressivité corporelle et mimique, la gestualité, n'ont pas de transcription écrite. Or, ici, il ne s'agit pas de transcription mais du passage d'une langue à l'autre car les langues signées ne disposent d'aucune forme écrite. L'exercice semble donc être encore plus complexe.

La commande du festival est d'ailleurs explicite puisque la programmation est constituée principalement de lectures publiques. Pas d'ambiguïté ici, les traducteurs opèrent dans une visée scénique. S'ajoute donc ici la performance de l'artiste lui-même. Baetens (2016) évoque la liberté grandissante que beaucoup de poètes prennent avec la version écrite du texte, voire le passage d'une lecture phonocentrique où l'on tente de reproduire sur scène le sens enfermé dans les mots sur la page à une logique du texte pluriel ou différentiel, c'est-à-dire une cuvre partagée entre plusieurs formes et versions équivalentes, radicalement ouvertes, toujours susceptibles de changement. Le traducteur est confronté à une triple difficulté : le changement de registre, le passage d'une langue à l'autre et la mouvance du texte au cours de la performance scénique. Cela pose des questions de traductologie pure, sur le processus de traduction et la qualité de la traduction en tant que produit fini, tout autant que sur la posture du traducteur.

\subsection{Un spectacle vivant}

61 Si la poésie signée pose une question de registre, elle pose aussi la problématique de la scénarisation, du jeu scénique de la déclamation en public d'un poème signé (en version originale ou issu d'une traduction). Certes, les traducteurs s'interrogent sur la limite entre verbal et non-verbal, mais ils ont conscience de personnifier le poème. En langue des signes, nous incarnons tout de suite le texte nous dit Marie Lamothe (entretien, 2017). Fidélité au texte et fidélité au poète priment. À aucun moment nous ne quittons le texte. La traduction adaptation que nous réalisons respecte le texte. Mais les traducteurs ne s'interdisent pas non plus la fidélité aux émotions.

62 À l'occasion de la journée d'études « Traduire l'intraduisible? Poésie et langue(s) des signes » organisée par l'université Paris 8 le 13 novembre 2015, Marie Lamothe, précise que la performance scénique est nécessairement prise en compte. La commande de départ est une traduction de poème, elle est donc appréhendée à partir de la forme écrite du texte. Mais cette traduction sera présentée en lecture publique. Lors du 
festival, les poèmes en français sont lus par des comédiens de langue française, ou par les auteurs eux-mêmes, qu'ils soient natifs du français ou non, face au public. Simultanément, les traducteurs, sur scène, signent leur traduction. Sachant cela, les traducteurs œuvrent en ayant en tête la scène.

Ce festival s'inscrit dans le refus de la dichotomie entre littératures orale et écrite. Il concrétise ce que Roubaud (2010) affirme :

La poésie doit se lire et se dire. Le poème doit être composé à la fois pour une page, pour une voix, pour une oreille, et pour une vision intérieure (Baetens, 2016).

L'oreille sera comprise, ici, comme l'œil pour les poèmes en langues signées. Les poètes Sourds se rallient également à ce point de vue, puisque nous l'avons observé, ils pensent leurs poèmes dans une visée scénarisée, soit en public, soit en vidéo. L'accointance entre la poésie signée et la scène est ici réelle et assumée.

Marie Lamothe reconnaît le caractère vivant de ses traductions en langue des signes, leur dimension inachevée :

J'arrête ma traduction sur scène. [...] J'ai tout préparé mais le jour $\mathrm{J}$, dans ma traduction, le verbe ne sera pas exactement au même endroit.

Les traducteurs ne livrent pas l'interprétation simultanée du discours prononcé à ce moment-là par le poète ou comédien, bien qu'ils s'appuient sur ce qu'ils entendent. Ils interprètent - au sens théâtral du terme - le résultat de leur traduction. Le traducteur devient ainsi le performeur de sa propre traduction. Certains sont à l'aise avec cette performance, d'autres non.

Lors des premières participations à Voix Vives, les interprètes-traducteurs montaient sur scène et produisaient face au public le fruit de leur traduction. Ils portaient en direct le résultat d'un travail réalisé en amont. Récemment, ils ont essayé de se détacher de la dimension scénique de la commande, en procédant à la traduction mais en essayant de céder leur place sur scène à des comédiens Sourds, qui s'approprient le texte signé et en donnent une interprétation (au sens théâtral). Pour des raisons organisationnelles, ce n'est pas toujours possible. Cependant, le fait qu'un tiers puisse s'approprier une version traduite d'un poème semble une étape importante dans l'évolution de la pratique de la traduction de la poésie signée. Nous assistons à la dissociation entre traduction et performance scénique de la traduction en tant que produit fini. Il est possible que dans cet espace de dissociation l'on trouve les éléments permettant de distinguer ce qui relève de la traduction à proprement parlé de ce qui relève de l'art scénique. Se trouve alors posée la question du support transmissible d'une traduction.

\subsection{La vidéo, une forme d'écriture?}

La question de la transmission et de la conservation des œuvres signées ne semble cependant pas mobiliser les communautés Sourdes. Alors que la littérature écrite regorge d'anthologies, et cela dans les langues vocales du monde entier, les locuteurs de langues signées ne réussissent pas encore à constituer de bibliothèques des savoirs et d'anthologie littéraires dans leur langue. Certaines anthologies existent mais demeurent des cas isolés (pour la France : Liennel en 1978, Les Mains Fertiles en 2015, la collection de DVD Poésignes publiés par l'association Signes mais qui ne comporte qu'une seule entrée depuis 2011). Le support de cette transmission est nécessairement vidéo. Il convient d'analyser les différentes valeurs que peuvent prendre les œuvres 
signées enregistrées au format vidéo, pour comprendre la nature même de ces œuvres et comprendre le travail de traduction mené à partir de celles-ci.

Pour réaliser une traduction, le traducteur, natif de la LSF ou non, s'attache à un support de départ. Dans le cadre de la poésie en langue des signes, sa forme est mouvante. Le traducteur peut disposer soit d'une version vidéo qui lui est destinée, soit d'un enregistrement en public. Levent Beskardes considère cependant que les enregistrements vidéo figent la forme de ses poèmes. On retrouve la logique du texte pluriel, que décrit Baetens (2016). Le poète poursuit, précisant que, d'une prise de vue à l'autre, suivant les sensations du corps et les mimiques du visage, la façon de rendre le temps, le soleil ou le vent varie. La vidéo ne peut pas être considérée comme un équivalent de la forme écrite ici, mais comme l'enregistrement d'une performance. Le traducteur, qu'il soit sourd ou entendant, doit opérer la bascule entre une forme vivante parmi d'autres, et une forme fixée par l'écrit. Il fige forcément sa traduction en la diffusant par écrit, en suivant les codes de l'écrit, même s'ils sont relativement libres en ce qui concerne la poésie contemporaine.

Dans l'autre sens, l'enregistrement vidéo d'une traduction en langue des signes à l'occasion d'une performance scénique gage encore moins d'une version figée. Le poème est donné au public et l'artiste module son expression en suivant ses propres aspirations et les réactions du public.

71 En 2015 est parue la première anthologie française de poésies signées, Les mains fertiles, 50 poètes en langue des signes, sous la direction de Brigitte Baumié. Cet ouvrage constitue la première trace écrite et vidéo, accessible au grand public, des traductions réalisées dans le cadre du festival de poésie "Voix vives, de Méditerranée en Méditerranée ». Il rassemble des poèmes en langue des signes dans leur version originale et des traductions de poésies créées en français mais aussi dans d'autres langues vocales. Toutes les vidéos [de traductions] n'ont pas le même statut. Certaines sont réalisées devant un fond noir, l'image est impeccable. C'est un produit fini. D'autres sont réalisées en direct pendant le festival.

Il existe une hiérarchie dans le statut des supports vidéo. D'une part, on observe la traduction filmée pour être figée dans une forme unique, léchée, et pour laquelle des moyens techniques particuliers sont mis en place. Cela correspond à un véritable dispositif d'écriture. D'autre part, il existe une forme vivante, mouvante, prise sur le vif. C'est l'image d'une forme orale. Les captations vidéo de l'interprétation en langue des signes de lectures de poésie au festival Voix Vives sont des traces figées, en tant que témoignage d'une oralité, d'une performance scénique. Elles illustrent la performance du comédien entendant, tout autant que celle du traducteur qui devient le temps de la lecture un comédien (qu'il le veuille ou non). Même s'il semble y avoir un parallèle entre une forme écrite figée et une forme vidéo figée, il n'est pas sûr qu'elles soient équivalentes.

\subsection{Quand le traducteur laisse la place au comédien}

73 Nous avons vu qu'il existe un lien entre la poésie signée et l'art scénique. Quelle est la nature de ce lien? Est-il à chercher dans le profil des poètes Sourds eux-mêmes? Nombre d'entre eux sont aussi comédiens: Levent Beskardes, Djenebou Bathily, Mathilde Chabbey ou François Brajou pour ne citer que certains d'entre eux. Leur aisance sur scène conditionne-t-elle leurs productions signées ou est-ce le contraire? 
Le lien entre poésie signée et art scénique serait plutôt à chercher dans la limite entre littérature signée et sign-art, pour reprendre l'expression de Pollitt (2014).

Lors de la lecture d'un poème, par exemple à Sète, le poète lit-il ou interprète-t-il, qu'il s'agisse de son propre poème ou de celui d'un autre? Le festival fait appel à des comédiens pour interpréter (au sens de «jouer ») les poèmes. Les poètes lisant leur propre texte colorent leur lecture, comme des comédiens. Nous ne sommes donc pas face à de l'écrit transcodé à l'oral mais face à une incarnation du texte à l'oral.

Qu'en est-il des interprètes (professionnels du passage entre LSF et français) quand ils présentent leur traduction? Carlos Carreras fournit des éléments de réponse :

C'est donc à nous de lui donner corps, sens et forme. C'est en ça que ce n'est pas une traduction comme les autres, dans la mesure où l'on incarne le texte, une œuvre, qui nécessite un travail sur la langue, le corps et l'esprit pour lui donner une autre forme (cité par Eckert-Poutot, 2016).

Si le traducteur incarne sa traduction, il est difficile de se figurer son absence, de se détacher de cette personnification du texte. Marie Lamothe ne dit pas autre chose quand elle qualifie d'interprétations les enregistrements réalisés en direct.

Les interprètes langue des signes française / français mobilisés dans ce cadre produisent-ils la traduction qu'ils ont préparée ? La version préparée à partir d'un écrit se confronte à la scène et son oralité. S'agit-il d'une traduction, ou de la préparation en vue de l'interprétation simultanée d'une performance de l'artiste parlant? Marie Lamothe relate l'expérience qu'elle a vécue avec la traduction d'un poème de Sapho, qu'elle avait préparée à partir du texte écrit. Lors de la lecture publique par Sapho ellemême, l'interprète s'est retrouvée en difficulté face à la prosodie de la poétesse, face à l'ajout de rires qui apportaient une connotation, qui changeaient le poème.

Dans ma traduction, cela ne changeait pas l'enchainement des gestes ou leur emplacement mais cela changeait l'émotion que j'avais prévue (entretien, 2017).

La traductrice décrit ici sa difficulté face à la performance de la poétesse, face à son jeu scénique. La forme de départ écrite du texte, fixée, laisse au lecteur la liberté d'infléchir sa lecture suivant son propre jeu scénique. La forme orale impose la forme scénique de celui qui déclame. Les réflexes professionnels de l'interprète le rendent conscient du décalage entre sa propre traduction du texte écrit et la lecture qu'en fait le poète. Mais malgré tout, le traducteur doit laisser la place au comédien et assumer cette bascule. La forme traduite est bien une œuvre en soi, du traducteur à partir de l'œuvre originale.

\section{L'œuvre traduite}

9 Le processus de traduction, quel qu'il soit, part toujours d'un texte source pour arriver à un texte cible, par le biais d'un traducteur, qu'il se réclame traducteur de profession ou non. Nous avons questionné le statut du texte source et celui du traducteur. Il est légitime de s'interroger sur le statut du produit fini, de l'œuvre traduite. L'œuvre traduite a-t-elle une existence autonome face au texte de départ?

Dans les langues disposant de forme écrite, la traduction en tant que produit fini est autonome, est libérée de son traducteur. Le lecteur accède à l'œuvre sans parfois se rendre compte qu'il s'agit d'une traduction, ou en l'oubliant partiellement le temps de sa lecture. En est-il de même pour les œuvres traduites en langues signées?

La démarche du traducteur nous éclaire pour comprendre le statut du « produit fini ».

TIPA. Travaux interdisciplinaires sur la parole et le langage, 34 | 2018 
Notre but est que le public ait accès au même texte mais dans deux langues différentes. Est-ce de la traduction littérale ou de l'adaptation? De l'adaptation! nous dit Marie Lamothe. La langue des signes est une langue qui a une puissance littéraire comme les langues orales. Il y a un aspect militant dans le fait d'avoir accepté de traduire des poésies. Il y a bien des poètes Sourds, la littérature Sourde existe. L'enjeu est là ! l'autre et non simultanément. Pour Marie Lamothe, aucun doute, œuvre de départ et
traduction sont des œuvres d'art toutes les deux. Carlos Carreras le confirme quand il décrit les traducteurs comme des poètes :

Nous sommes tous poètes, et certains interpoètes! (cité par Eckert-Poutot, 2016).

Il replace donc bien, lui aussi, le produit de la traduction comme une version autonome, une œuvre en soi, qui suit un chemin parallèle à l'œuvre originale. Notons qu'une traduction n'est, en soi, jamais achevée mais que le traducteur l'arrête bel et bien, tel un auteur qui arrête son texte à une version donnée pour publication. Cependant, on n'assiste pas encore à la diffusion de ces traductions en français indépendamment de leur original en langue des signes. Leur diffusion s'opère toujours dans un cadre bilingue et jamais monolingue.

\section{BIBLIOGRAPHY}

Baetens, J. (2016) À voix haute : poésie et lecture publique, Bruxelles: Les Impressions nouvelles (Réflexions faites).

Bahan, B. (2008) Upon the formation of a visual variety of the human race, in Bauman, H-D. L. (ed.) Open your eyes: deaf studies talking, Minneapolis: University of Minnesota Press.

Baumié, B. (2017) Poésie en langue des signes, une expérience de la rencontre de la parole et du corps [en ligne]. Disponible sur : https://www.terreaciel.net/Poesie-en-langue-des-signes-une-experiencede-la-rencontre-de-la-parole-et-du\#.W1iFr_ZuKUk (consulté le 25 juillet 2018).

Blondel, M. (2000) Poésie enfantine dans les langues des signes : modalité visuo-gestuelle versus modalité audio-orale, Thèse de doctorat, Université de Tours.

Bouvet, D. \& M.-A. Morel (2002) Le ballet et la musique de la parole, Paris: Ophrys.

TIPA. Travaux interdisciplinaires sur la parole et le langage, 34 | 2018 
Chateauvert, J. (2014) Poétique du mouvement : ce que les langues des signes font à la littérature [en ligne], Thèse de doctorat, Université du Québec, Montréal. Disponible sur : http://poetiquels.net/ (consulté le 25 juillet 2018).

Cormier, K. (2014) Pronouns, agreement and classifiers: What sign languages can tell us about linguistic diversity and linguistic universals, in UCL Working Papers in Linguistics, 26, p. 1-12.

Delaporte, Y. (1999) Les Sourds c'est comme ça, Paris: Editions de la Maison des Sciences de l'Homme.

Eckert-Poutot, E. (2016) L'interpoète, Le journal de l'AFILS, 92, p. 13-15.

Ellrodt, R. (2006) Comment traduire la poésie ?, Palimpsestes, hors-série, p. 65-75.

Fonagy, I. (1983) La vive voix, Essai de psycho-phonétique, Paris: Payot.

Frishberg, N. (1988) Signers of tales, in Sign Language Studies, Gallaudet University Press.

Guaïtella, I. (2013) Le langage sans frontières, Nouvelles approches pour l'étude de la communication, Paris: L'Harmattan.

Genette, G. (1982) Palimpsestes. La littérature au second degré, Paris: Seuil.

Goldin-Meadow, S. (2003) Hearing gesture: how our hands help us to think, Cambridge: Harvard University Press.

Huyghe, R. (1955) Dialogue avec le visible, Paris: Flammarion.

Kendon, A. (2008) Some reflexions on the relationship between 'gesture' and 'sign', Gesture, 8, 3, Benjamins Pub., p. 348-366.

Liennel, C. (1978) Les Voix Invisibles, Recueil de seize poèmes, Vincennes: Centre Socio Culturel des sourds-IVT.

Lillo-Martin, D. (2012) Utterance report and constructed action, in Pfau, R., Steinbach, M. \& B. Woll (eds) Sign Language - An international Handbook, Berlin: Walter de Gruyter, p. 365-387.

Mottez, B. (1993) Les Sourds existent-ils ?, Revue du collège des Psychanalystes, 46-47, p. 49-58.

Mottez, B. (1987/2003) L'identité Sourde, Les Sourds existent-ils ? Textes rassemblés et présentés par Andrea Benvenuto, Paris: L'Harmattan, p. 64-85.

Peeters, J. (1999) La médiation de l'étranger : une sociolinguistique de la traduction, Arras : Artois Presses Université.

Pollitt, K.M. (2014) Signart: (British) sign language poetry as Gesamtkunstwerk, Thèse de doctorat, University of Bristol.

Risler, A. (2016a) Langues des signes le corps en jeu : présence, absence, distance, L'Information Grammaticale, 149 , p. 2-6.

Risler, A. (2016b) Parler de soi, parler des autres en LSF, L'Information Grammaticale, 149, Ophrys, p. $45-54$.

Rose, H. (1992) A semiotic analysis of artistic American Sign Language and a performance of poetry, Text and Performance Quarterly, 12, p. 146-159.

Roubaud, J. (2010) Obstination de la poésie, Le Monde diplomatique, janvier 2010, p. 22-23.

Schetrit, O. (2013) Dépasser la violence par la création ?, Anthrovision [En ligne], 1, 2. Disponible sur : http://journals.openedition.org/anthrovision/569 (consulté le 25 juillet 2018). 
Schetrit, O. (2009) Chansignes et chorésignes, la théatralisation chorégraphique de la contestation sociale des personnes sourdes, in Conférence au musée du Quai Branly, 1er décembre 2009, Paris.

Sutton-Spence, R. (2005) Analysing sign language poetry, New York: Palgrave Macmillan.

Sutton-Spence, R. \& M. Kanedo (2007) Symmetry in sign language poetry, Sign Language Studies, 7, p. 284-318.

Taub, S. (2001) Language from the Body: Iconicity and Metaphor in American Sign Language, Cambridge : Cambridge University Press.

Thion, M. (2015) Les Mains Fertiles, Paris : Editions Bruno Doucey.

Valli, C. (1993) The Poetics of American Sign Language Poetry, Thèse de doctorat, The Union Institute Graduate School.

\section{APPENDIXES}

\section{Filmographie}

Fino-Bergé, S. (2013) Tout un poème, film réalisé pour L'Æil et la Main, France 5 https://www.france.tv/france-5/1-oeil-et-la-main/51401-tout-un-poeme.html

Hikmet, N. (1958) Légendes des légendes, traduction en langue des signes turque de L. Beskardes, https://www.youtube.com/watch?v=bvgOMI7Y0yg

Hikmet, N. Mes funérailles, traduction en langue des signes française de L. Beskardes, https://www.youtube.com/watch?v=cRPVLaR3poE

Mako, A. (2013), Portrait n³ - Djenebou Bathily, entretien réalisé pour Bête à Bon Dieu Production - http://www.dailymotion.com/video/x15rva6

Ochronowicz, I. (2009) Sourds et malentendus, production Point du Jour, $70 \mathrm{~min}$.

\section{Entretiens}

Beskardes, L. Entretien avec P. Houwenaghel, 17 août 2017

Markowicz, A. La traduction est un exercice de reconnaissance, de gratitude envers l'autre, émission La Grande table, France Culture, 28 octobre 2015.

Lamothe, M. Entretien avec P. Houwenaghel, 22 août 2017.

\section{NOTES}

1. Les termes utilisés ici en français de jeu corporel et prise de rôle renvoient à la distinction proposée en anglais par Lillo-Martin (2012) entre role play et role shift.

2. Le terme « chansigne » est initialement créé en langue des signes par les comédiens de Miracle par Hasard. Il est issu de la contraction des signes "chant » et "langue des signes » en un seul geste. Il a ensuite été traduit en français par chansigne ou chantsigne.

3. Première compagnie de théâtre française composée d'artistes Sourds, créée en 1976 par Jean Grémion et Alfredo Corrado. Elle jouera un rôle majeur dans le déploiement de la culture Sourde en France et la promotion de la langue des signes, notamment en mettant en scène des pièces en 
langue des signes et en proposant des cours de LSF et des ateliers d'initiation aux pratiques scéniques pour les Sourds.

4. La VHS ou video home system est un standard d'enregistrement de vidéo sur bande magnétique, créé par la société JVC en 1976.

5. Dans notre exemple, il s'agit d'une transcription signe à mot.

6. Ce poème fait partie d'un recueil de seize poèmes de Chantal Liennel, édité par le centre socioculturel des Sourds-IVT en 1978.

7. Olivier Schétrit évoque ce poème lors d'une conférence en 2009 ( Schétrit, 2009); un extrait vidéo de Fleur est également disponible en ligne (Schétrit 2013).

8. Voir le portrait de la poétesse réalisé par Mako (2013).

9. Le CETIM, Centre de Traduction, d'Interprétation et de Médiation Linguistique, rattaché à l'Université Jean Jaurès de Toulouse, propose une licence de traduction et médiation français/ LSF/anglais et un master de traduction et interprétation français-anglais-LSF spécifiquement accessibles aux étudiants sourds.

10. Poème visible dans le documentaire de Sophie Fino-Bergé pour l'émission L'đFil et la Main, diffusé en 2013.

\section{ABSTRACTS}

Recent studies on poetry in signed languages focus on the classification of literary material and the identification of stylistic features used by poets. Here, our aim is to go further and try to understand what signed poetry and its translation reveal about signed languages. Translation raises an issue of inter-cultural barriers. As Newmark (1981) said, translation is part of intellectual life of every civilized people. The community of the Deaf is not an exception. The translations are imported into and exported from its cultural territory. Poetry, this cultural material, leads us to examine the question of translation with the perspective of a text's transition from one culture to another.

Studies in linguistics of sign language face a problem for setting boundaries between verbal, nonverbal and gestural components of a signer's expression. What can be designated as a "text", in a signed language? In terms of narratives, it is not easy to answer this question. But it is even more complex in the performance poetry in signed language, where verbal, corporal, spatial, facial expression are very closely coupled. The process of translation of poetry between French and French sign language forces us to consider a double process: on the one hand, from one language and culture into another, and on the other hand, from oral to written style and vice versa.

What do translators actually do? Who are the translators?

Different methods and ways of translating between French and FSL poems have been experimented: a deaf poet who translates by himself because he has bilingual and bicultural abilities; an outside source who translates with no connection to the poet; the pairing of a deaf poet and a hearing sign language interpreter; or a cross functional translation team composed by deaf and hearing poets, and deaf and hearing translators.

These translation proceedings give the opportunity to consider the sense of the word "interpreter" in French. Professional sign language interpreters are well placed to be part of such a translating team. Their professional abilities consist of switching from one language into another. But in French, the word "interpreter" has different meanings. It is also equivalent to "performer". Is the interpreter the only performer of his or her work? In other words, do 
translated poems have an independent existence? Also, is it possible to consider a signed poem independently from its author? Can we consider video recording as a written form? Finally, how can one share a signed poem with the public?

Our presentation is based, on one hand, on the experience of two deaf poets, who both compose poems in FSL and are involved in different translation proceedings: Levent Beskardes and François Brajou; and, on the other hand, on the experience of French/FSL interpreters who are particularly interested and involved in the translation of signed poetry. Together, they will participate in a workshop preparing a poetry festival (Voix Vives, held in the city of Sete, France). During this festival, poems and their translations are performed live. This allows us to examine the relations between signed poetry and stage performance.

Les premières recherches portant sur la poésie signée sont relativement récentes et s'attachent à classifier les œuvres et à observer les effets stylistiques produits par les poètes. Nous tenterons de dépasser ces questions pour essayer de comprendre ce que la poésie signée, et la traduction de ces œuvres, nous dit des langues signées en général. Les recherches linguistiques peinent encore à délimiter la part verbale, non-verbale et para-verbale dans l'expression des signeurs. La tâche se révèle encore plus ardue face à la performance poétique signée, qui dépasse largement les frontières du texte. Dès lors, comment comprendre le travail des traducteurs? Comment opèrent-ils ? Comment passer d'un texte à une forme signée et vice-versa ? Les travaux de poètes, d'interprètes et de traducteurs éclaireront notre réflexion et nous amèneront à questionner la forme même de la poésie signée en tant que performance scénique.

\section{INDEX}

Mots-clés: langue des signes, poésie, traduction, interprétation, performance Keywords: signed language, poetry, translation, interpretation, performance

\section{AUTHORS}

\section{PÉNÉLOPE HOUWENAGHEL}

Université de Lille, France

penelope.houwenaghel@free.fr

\section{ANNIE RISLER}

Savoirs, Textes, Langage - CNRS \& Université de Lille, France

annie.risler@univ-lille.fr 